Ophthalmologe 2017 $\cdot 114: 272$

DOI 10.1007/s00347-017-0443-9

Online publiziert: 30. Januar 2017

(c) Springer Medizin Verlag GmbH 2017

CrossMark

\section{Leserbrief zu}

Duisdieker V, Loeffler KU et al (2016)

Transplantatversagen? Ophthalmologe

113:955-956, doi: http://dx.doi.org/10.1007/ s00347-016-0254-4

Die Autoren beschreiben bei einem 50jährigen Patienten, bei dem jeweils 1996 am rechten und 2005 am linken Auge eine perforierende Keratoplastik bei Keratokonus durchgeführt wurde, ein Keratokonusrezidiv rechts. Nach 18 Jahren trat am rechten Auge in der inferioren Transplantathälfte ein Epithelödem mit Descemet-Falten auf. Durch die partielle Hornhauttrübung verschlechterte sich der Visus rechts auf 0,1 ; links betrug er 0,8 .

Eine lokale und eine systemische Kortisontherapie blieben erfolglos. Erst eine zweite perforierende Keratoplastik rechts brachte die klare Einheilung des $8 \mathrm{~mm}$ großen Transplantates.

Histologisch zeigte das entnommene Hornhautscheibchen neben der Hornhautverdickung durch das Ödem keratokonustypische Unterbrechungen der Bowman-Membran.

Dieser Befund widerspricht der Abstoßungsreaktion.

Die Autoren geben verschiedene Theorien für die Entstehung des Keratokonusrezidivs an:

- Das Transplantat wird von Keratozyten des Empfängers infiltriert.

- Enzymatische Veränderungen des

Empfängerepithels oder

Eine Erwiderung zu diesem Leserbrief finden Sie unter doi: http://dx.doi.org/10.1007/s00347017-0442-x

\title{
M. Jähne
}

Klinik für Augenheilkunde, Klinikum Chemnitz, Chemnitz, Deutschland

\section{Keratokonusrezidiv durch zu kleines Transplantat}

\author{
- subklinischer Keratokonus in der \\ Spenderhornhaut?
}

Bei genauer Betrachtung der Abb. 1 in der Publikation sieht man, dass das Rezidiv des Keratokonus sich vorwiegend auf den nasal unteren Rand des relativ kleinen Transplantates lokalisiert. So ist das klinische Bild auch beim fortgeschrittenen bzw. akuten Keratokonus - die Spitze ist gering nach nasal unten dezentriert.

Nimmt man einen Stechzirkel zur Hand und ordnet dem Hornhautdurchmesser etwa $11 \mathrm{~mm} \mathrm{zu}$, dann ist das Transplantat der ersten Keratoplastik von 1996 mit 6,0 bzw. 6,2 mm für eine perforierende Keratoplastik beim Keratokonus eindeutig zu klein gewesen. Damit geht die Pathogenese für das Keratokonusrezidiv im Transplantat von der Wirtskornea aus.

Schon 1974 konnte der Verfasser einen solch seltenen Kasus beobachten und publizieren [1]: Bei einem 10-jährigen Knaben mit Keratokonus wurde in der Augenklinik Dresden-Johannstadt 1951 ein 4-mm-Transplantat nur mit Bindehautdeckung befestigt. 1973 Verschlechterung durch Keratokonusrezidiv. Klare Einheilung des nun 8,5 mm großen Transplantates erst nach zweiter Rekeratoplastik. Histologische Untersuchungen waren 1973 an der Zittauer Augenklinik noch nicht möglich.

Klinische Studien noch in den 70erJahren zeigten, dass kleine Transplantate bis $6 \mathrm{~mm}$ gut einheilten, aber meist nicht für die vollständige Abflachung des Konus ausreichten.

Bei unserem späteren Patientengut an der Augenklinik in Aue/Sachsen fanden wir unter 642 perforierenden Keratoplastiken 96 Augen mit Keratokonus (15\%). In der Mehrzahl der Fälle kamen die
Trepangrößen von 7,0-8,0 mm mit einer Überdimensionierung des Transplantates bis $0,2 \mathrm{~mm}$ zum Einsatz. Nie mehr konnten wir ein Keratokonusrezidiv im Transplantat beobachten $[2,3]$.

\section{Korrespondenzadresse}

PD Dr. M. Jähne, FEBO

Klinik für Augenheilkunde, Klinikum Chemnitz Flemmingstr. 2, 09116 Chemnitz, Deutschland dr.m.jaehne@t-online.de

Interessenkonflikt. M. Jähne gibt an, dass kein Interessenkonflikt besteht.

\section{Literatur}

1. Jähne M (1974) Keratokonusrezidiv nach Keratoplastik. ZÄrztl Fortbild 68(9):434-436

2. Jähne M (2002) Perforierende Keratoplastik beim Keratokonus. Augenspiegel 48(3):8-14

3. Jähne M (2006) Akuter Keratokonus - abwarten oder früher operieren? Ophthalmol Nachr 3:10-11 